\title{
The Evolving Role of Succinate in Tumor Metabolism: An ${ }^{18}$ F-FDG-Based Study
}

\author{
Philippe Garrigue ${ }^{1-3}$, Aurore Bodin-Hullin ${ }^{3}$, Laure Balasse ${ }^{1,2}$, Samantha Fernandez ${ }^{2}$, Wassim Essamet ${ }^{4}$, \\ Françoise Dignat-George ${ }^{1}$, Karel Pacak ${ }^{5}$, Benjamin Guillet ${ }^{1-3}$, and David Taïeb ${ }^{2,3}$ \\ ${ }^{1}$ Aix-Marseille University, INSERM, UMR-S 1076, Marseille, France; ${ }^{2}$ Aix-Marseille University, CERIMED, Marseille, France; \\ ${ }^{3}$ Department of Nuclear Medicine, Aix-Marseille University, Marseille, France; ${ }^{4}$ Department of Neuropathology, APHM Timone, \\ Marseille, France; and ${ }^{5}$ Section on Medical Neuroendocrinology, Eunice Kennedy Shriver National Institute of Child Health and \\ Human Development (NICHD), National Institutes of Health, Bethesda, Maryland
}

\begin{abstract}
In recent years, inherited and acquired mutations in the tricarboxylic acid (TCA) cycle enzymes have been reported in diverse cancers. Pheochromocytomas and paragangliomas often exhibit dysregulation of glucose metabolism, which is also driven by mutations in genes encoding the TCA cycle enzymes or by activation of hypoxia signaling. Pheochromocytomas and paragangliomas associated with succinate dehydrogenase (SDH) deficiency are characterized by high ${ }^{18} \mathrm{~F}-\mathrm{FDG}$ avidity. This association is currently only partially
\end{abstract} explained. Therefore, we hypothesized that accumulation of succinate due to the TCA cycle defect could be the major connecting hub between SDH-mutated tumors and the ${ }^{18} \mathrm{~F}-\mathrm{FDG}$ uptake profile. Methods: To test whether succinate modifies the ${ }^{18} \mathrm{~F}-\mathrm{FDG}$ metabolic profile of tumors, we performed in vitro and in vivo (smallanimal PET/CT imaging and autoradiography) experiments in the presence of succinate, fumarate, and phosphate-buffered saline (PBS) in different cell models. As a control, we also evaluated the impact of succinate on ${ }^{18} \mathrm{~F}$-fluorocholine uptake and retention. Glucose transporter 1 (GLUT1) immunohistochemistry was performed to assess whether ${ }^{18} \mathrm{~F}-\mathrm{FDG}$ uptake correlates with GLUT1 staining. Results: Intratumoral injection of succinate significantly increased ${ }^{18} \mathrm{~F}-\mathrm{FDG}$ uptake at $24 \mathrm{~h}$ on small-animal PET/CT imaging and autoradiography. No effect of succinate was observed on cancer cells in vitro, but interestingly, we found that succinate caused increased ${ }^{18}$ F-FDG uptake by human umbilical vein endothelial cells in a concentration-dependent manner. No significant effect was observed after intratumoral injection of fumarate or PBS. Succinate, fumarate, and PBS have no effect on cell viability, regardless of cell lineage. Intramuscular injection of succinate also significantly increases ${ }^{18} \mathrm{~F}$ FDG uptake by muscle when compared with either PBS or fumarate, highlighting the effect of succinate on connective tissues. No difference was observed between PBS and succinate on ${ }^{18} \mathrm{~F}$-fluorocholine uptake in the tumor and muscle and on hind limb blood flow. GLUT1 expression quantification did not significantly differ between the study groups. Conclusion: The present study shows that succinate stimulates ${ }^{18} \mathrm{~F}-\mathrm{FDG}$ uptake by endothelial cells, a finding that partially explains the ${ }^{18} \mathrm{~F}-\mathrm{FDG}$ metabotype observed in tumors with $\mathrm{SDH}$ deficiency. Although this study is an ${ }^{18} \mathrm{~F}-\mathrm{FDG}$-based approach, it provides an impetus to better characterize the determinants of ${ }^{18} \mathrm{~F}-\mathrm{FDG}$ uptake in various tumors and their surrounding microenvironment, with a special emphasis on the role of tumor-specific oncometabolites.

Received Mar. 6, 2017; revision accepted May 24, 2017.

For correspondence or reprints contact: David Taïeb, Department of Nuclear Medicine, La Timone University Hospital, European Center for Research in Medical Imaging, Aix-Marseille University, 264 rue Saint-Pierre, 13385 Marseille, France.

E-mail: david.taieb@ap-hm.fr

Published online Jun. 15, 2017.

COPYRIGHT (C 2017 by the Society of Nuclear Medicine and Molecular Imaging.
Key Words: succinate; ${ }^{18} \mathrm{~F}-\mathrm{FDG}$; succinate dehydrogenase; paraganglioma; tricarboxylic acid cycle

J Nucl Med 2017; 58:1749-1755

DOI: 10.2967/jnumed.117.192674

$\mathbf{F}$ or many decades, succinate (succinic acid in blood $\mathrm{pH}$ ) has been considered only an intermediate metabolite of the tricarboxylic acid (TCA) cycle. During aerobic respiration, succinate is oxidized to fumarate, donating reducing equivalents. The reaction is catalyzed by succinate dehydrogenase (SDH), an enzyme complex located in the inner mitochondrial membrane that participates in both the TCA cycle and the electron transport chain. SDH comprises 4 nuclearly encoded subunits whose structure and genes have mostly been conserved through evolution. The Hans Adolf Krebs team noticed that some intermediates, including succinate, could accumulate in the interstitial space during liver ischemia (1). During ischemia, succinate can be produced by reduction of fumarate (a purine nucleotide cycle metabolite) via the reverse action of SDH. Succinate is then secondarily secreted from the cells into the bloodstream (2). Many studies have shown that succinate has several functions beyond participating in the TCA cycle, of which some are mediated via a $G$ protein-coupled succinate receptor (GPR91) (3). Through GPR91, succinate may have hormonelike actions in blood cells, as well as in fat, liver, heart, retina, and kidney tissues (4). For instance, in response to retinal ischemia, succinate plays an important role in the development of new blood vessels via GPR91 and subsequent modulation of vascular endothelial growth factor release by retinal ganglion neurons (5).

Beyond the cell functions described above, succinate and a few other TCA cycle intermediates were found to contribute to carcinogenesis (6). Recently, germline and somatic mutations in an additional 3 TCA cycle enzymes-fumarate hydratase, malate dehydrogenase type 2 , and isocitrate dehydrogenase-were identified in diverse cancers, suggesting that metabolic alterations are the underlying hallmark of cancer. These mutations cause disruption of the TCA cycle and accumulation of TCA cycle intermediates, ultimately altering various functions and the epigenome of cancer cells. These so-called oncometabolites were found to act as competitors of 2-oxoglutarate-dependent dioxygenases, which are involved in a broad spectrum of pathways such as hypoxic 
response, immune system dysfunction, and epigenetic reprogramming (7).

Pheochromocytomas and paragangliomas (PPGL) are tumors associated with TCA cycle defects (8). The most common cause of hereditary PPGL is SDH deficiency and accumulation of highly elevated levels of succinate. These tumors, unlike isocitrate dehydrogenase-mutant tumors (9), are highly glucose-avid (8). This metabolic pattern has been demonstrated by ${ }^{18} \mathrm{~F}$-FDG PET imaging studies (10). This finding is attributed to activation of hypoxia signaling (8) and is in discordance with several experimental studies that have failed to identify increased glycolysis (11-15). Interestingly, neuroblastoma cell lines (a neural-crest tumor model similar to PPGL) with SDHB mutations were even found to have a paradoxic decrease in glucose uptake compared with wild-type cells, despite an increased growth rate and invasiveness (16). These effects were more pronounced in the presence of human fibroblasts in coculture experiments, indicating a possible metabolic cooperation between stromal and cancer cells (17). Primary human fibroblasts exhibit an increased glucose uptake when they are cocultured with wild-type cells, and an even greater uptake when cocultured with $S D H B$-silenced neuroblastoma cell lines. Several studies have shown that yeast with $s d h \Delta$ mutations may aberrantly efflux succinate from the mitochondria (18-20) and that succinate is therefore likely to act as an extracellular ligand. This efflux of succinate is also presumed in humans because $\mathrm{SDH}$ related PPGL patients have a higher plasma succinate-to-fumarate ratio than patients with apparently sporadic disease and neurofibromatosis type $1(21)$. Therefore, we hypothesized that succinate could be the connecting hub between $S D H$ deficiency and the tumor ${ }^{18}$ F-FDG uptake profile via paracrine action on stromal cells.

\section{MATERIALS AND METHODS}

\section{Cells}

Human umbilical vascular endothelial cells (HUVECs) are commonly used as a laboratory model for studying the pathophysiology of endothelial cells (22) and tumor-stroma interactions (23). HUVECs were cultured in T175 plates in endothelial growth medium-2 (Lonza) supplemented with $10 \%$ decomplemented fetal calf serum at $37^{\circ} \mathrm{C}$ under $5 \% \mathrm{CO}_{2}$.

HT-29 is a human colorectal adenocarcinoma-derived cell line (obtained from a primary tumor). These cells were chosen because they have been fully characterized from an oncogenetic, metabolomics, and functional imaging standpoint. They are characterized by absence of succinate accumulation (24), a moderate avidity for ${ }^{18} \mathrm{~F}$ FDG $(25,26)$, and an activation of the mitogen-activated protein kinase pathway due to $B R A F$ mutations (27). HT-29 cells were cultured in T175 plates in Dulbecco modified Eagle medium (Lonza) supplemented with $10 \%$ fetal calf serum at $37^{\circ} \mathrm{C}$ under $5 \% \mathrm{CO}_{2}$.

Primary human cardiac fibroblasts (PromoCell) were cultured in T175 plates in fibroblast growth medium 3 (PromoCell) supplemented with $10 \%$ fetal calf serum at $37^{\circ} \mathrm{C}$ under $5 \% \mathrm{CO}_{2}$.

\section{In Vitro ${ }^{18}$ F-FDG Uptake by Endothelial Cells, Tumor Cells, and Fibroblasts}

HT-29 cells, HUVECs, and primary human cardiac fibroblasts were transferred to 6-well flasks and pretreated for $24 \mathrm{~h}$ with $0.01,0.1,1.0$, or $10 \mathrm{nmol}$ of succinate (Sigma-Aldrich) or fumarate (Sigma-Aldrich) per microliter of culture medium. Fumarate or succinate solutions were prepared at $1 \mathrm{mM}, \mathrm{pH} 7.4$, in phosphate-buffered saline (PBS). HT-29 cells allowed us to overcome the potential local self-secretion of succinate by tumor cells that could prevaricate any exogenous succinate impact on ${ }^{18} \mathrm{~F}-\mathrm{FDG}$ tissue uptake. PBS was used as a control. Each condition was repeated in triplicate. ${ }^{18} \mathrm{~F}-\mathrm{FDG}$ was added ( $2 \mathrm{MBq} /$ well) for $20 \mathrm{~min}$, and the cells were then washed 3 times with PBS, lysed with $0.2 \mathrm{M} \mathrm{NaOH}$, and counted on a COBRA-2 Auto Gamma Counter (Packard). The counting results were corrected by physical decay of ${ }^{18} \mathrm{~F}$ and expressed as mean-normalized ${ }^{18} \mathrm{~F}-\mathrm{FDG}$ uptake.

\section{In Vitro Evaluation of Cell Viability}

Cell viability was assessed by counting with trypan blue on Kova slides (Kova International) after a 24-h incubation with fumarate or succinate $(0.01$ or $10 \mathrm{nmol} / \mu \mathrm{L})$ and then compared with PBS treatment. The counting results were expressed as mean normalized number of viable cells.

\section{Mice}

All procedures using animals were approved by the institution's Animal Care and Use Committee (CE14, Aix-Marseille Université) and were conducted according to European Union Directive 2010/63/ EU and the recommendations of the Helsinki Declaration. Six-weekold BALB/c mice $(n=12)$ and Hsd:Athymic Nude-Foxn $1^{n u}$ mice $(n=15)$ were purchased from Envigo. The animals were housed in cages enriched with hay agglomerates and cocoons, placed in a temperature- and hygrometry-controlled room with daily monitoring, and given water and a commercial diet ad libitum.

\section{Xenograft Model}

HT-29 cells $\left(10^{7}\right)$ were trypsinized and resuspended in $500 \mu \mathrm{L}$ of complete medium (Dulbecco modified Eagle medium, $10 \%$ fetal calf serum, a $100 \mathrm{U} / \mathrm{mL}$ concentration of penicillin, and $100 \mu \mathrm{L}$ of Matrigel Basement Membrane Matrix High Concentration [Corning]). The animals were then allowed to rest for 2 wk. A first group of 3 Hsd: Athymic Nude-Foxn $1^{n u}$ mice was subcutaneously injected with $10^{6}$ HT-29 cells/100 $\mu \mathrm{L}$ on the right and left shoulders and on the right flank. A second group of $6 \mathrm{Hsd}$ :Athymic Nude-Foxn $1^{n u}$ mice was subcutaneously injected with $10^{6}$ HT-29 cells/100 $\mu \mathrm{L}$ on the right shoulder. A third group of $6 \mathrm{Hsd}$ :Athymic Nude-Foxn $1^{n u}$ mice was subcutaneously injected with $10^{6}$ HT-29 cells $/ 100 \mu \mathrm{L}$ on the right shoulder.

\section{In Vivo ${ }^{18}$ F-FDG Uptake After Intratumoral Injection of Succinate}

Fourteen days after tumor engraftment, the xenografted mice from the first group were injected with $10 \mu \mathrm{L}$ of a $1 \mathrm{nmol} / \mu \mathrm{L}$ succinate solution in the right shoulder tumor, with $10 \mu \mathrm{L}$ of a $1 \mathrm{nmol} / \mu \mathrm{L}$ fumarate solution in the left shoulder tumor, and with $10 \mu \mathrm{L}$ of PBS in the right hind limb tumor every $6 \mathrm{~h}$ for $24 \mathrm{~h}$ under $1.5 \%$ isoflurane anesthesia $(n=3) .{ }^{18} \mathrm{~F}-\mathrm{FDG}$ injection $(5-10 \mathrm{MBq} / 50 \mu \mathrm{L}$, intraperitoneally) was performed $3 \mathrm{~h}$ after the last succinate injection.

PET images were acquired $40 \mathrm{~min}$ after injection on a Mediso NanoPET/CT scanner under $1.5 \%$ isoflurane anesthesia. Region-ofinterest quantification was performed on reconstructed PET/CT images, corrected by a tumor volume, and weighted by animal weight.

At the end of the procedure, the animals were euthanized with a lethal dose of pentobarbital, and the tumors were then explanted, stored in $4 \%$ paraformaldehyde, sliced, and directly exposed for $30 \mathrm{~s}$ to medium-sensitivity phosphor imaging plates. Signals were analyzed by densitometry using Cyclone Plus (Perkin-Elmer). Image analysis and quantifications were performed on OptiQuant 5.0 software (Perkin-Elmer), and the results were expressed in digital light units $/ \mathrm{mm}^{2}$ as mean $\pm \mathrm{SD}$.

\section{In Vivo Dynamic ${ }^{18}$ F-FDG and ${ }^{18}$ F-Fluorocholine Uptake After Intratumoral Injection of Succinate}

Fourteen days after tumor engraftment, the xenografted mice from the second group were injected with a $1 \mathrm{nmol} / \mu \mathrm{L}$ succinate solution or 
with PBS ( $10 \mu \mathrm{L}$ in the tumor, $n=3$ per condition) every $6 \mathrm{~h}$ for $24 \mathrm{~h}$ under $1.5 \%$ isoflurane anesthesia. ${ }^{18} \mathrm{~F}-\mathrm{FDG}$ injection $(5-10 \mathrm{MBq} / 50 \mu \mathrm{L}$, intravenously) was performed $3 \mathrm{~h}$ after the last succinate injection. The xenografted mice from the third group were injected with a $1 \mathrm{nmol} / \mu \mathrm{L}$ succinate solution or with PBS $(10 \mu \mathrm{L}$ in the tumor, $n=3$ per condition), every $6 \mathrm{~h}$ for $24 \mathrm{~h}$ under $1.5 \%$ isoflurane anesthesia. ${ }^{18} \mathrm{~F}$-fluorocholine injection $(5-10 \mathrm{MBq} / 50 \mu \mathrm{L}$, intravenously) was performed $3 \mathrm{~h}$ after the last succinate injection. PET images were acquired beginning with the injection on a Mediso NanoPET/CT camera under $1.5 \%$ isoflurane anesthesia. PET images were reconstructed in dynamic mode with 10 frames of $1 \mathrm{~min}$ and then 6 frames of $5 \mathrm{~min}$ followed by one 20-min frame. Region-of-interest quantification was performed on PET/CT images, corrected by a tumor volume.

\section{In Vivo Dynamic ${ }^{18}$ F-FDG and \\ ${ }^{18}$ F-Fluorocholine Uptake After Intramuscular Injections of Succinate}

Twelve BALB/c mice were injected in the right quadriceps femoris muscle with succinate $(10 \mu \mathrm{L}$ of a $1 \mathrm{nmol} / \mu \mathrm{L}$ solution $)$ and in the left quadriceps femoris muscle with fumarate $(10 \mu \mathrm{L}$ of a $1 \mathrm{nmol} / \mu \mathrm{L}$ solution, $n=6)$ or with PBS $(n=6)$.

${ }^{18} \mathrm{~F}-\mathrm{FDG}$ and ${ }^{18} \mathrm{~F}$-fluorocholine injections (5-10 MBq/50 $\mu \mathrm{L}$, intravenously) were performed on different groups $3 \mathrm{~h}$ after the last succinate injection. PET images were acquired beginning with the injection on a Mediso NanoPET/CT camera under $1.5 \%$ isoflurane anesthesia. PET images were reconstructed in dynamic mode with 10 frames of $1 \mathrm{~min}$ and then 6 frames of $5 \mathrm{~min}$ followed by one 20-min frame.

Laser-Doppler perfusion imaging (Perimed) was used to assess hind limb blood flow as previously described (28), immediately after the ${ }^{18} \mathrm{~F}-\mathrm{FDG}$ acquisition ( $n=3$ per condition). The results were expressed as a ratio of blood flow in the succinate-treated limb to that in the PBS- or fumarate-treated limb.

\section{Immunohistochemistry}

To assess whether the increased uptake was due to glucose transporter 1 (GLUT1) overexpression after succinate treatment, GLUT1 immunohistochemistry (GLUT1, rabbit polyclonal antibody [catalog number RB-9052-P; Thermo Scientific]; dilution, 1:200) was performed on HT-29 tumors and HUVECs treated with succinate, fumarate, or PBS. The immunoreactivity of GLUT1 was visually scored by a pathologist masked to the study groups.

\section{Statistics}

Comparison of in vitro cellular uptake and cell viability was analyzed by 1 -way ANOVA with post hoc Bonferroni testing. In vivo uptake of ${ }^{18} \mathrm{~F}$-FDG and ${ }^{18} \mathrm{~F}$-fluorocholine in muscles and tumors and laser-Doppler data were compared by 2-tailed Mann-Whitney testing. A $P$ value of less than 0.05 indicated statistical significance.

\section{RESULTS}

Succinate Increases Tumor ${ }^{18}$ F-FDG Uptake and Retention In Vivo

To test whether succinate modifies the ${ }^{18} \mathrm{~F}-\mathrm{FDG}$ metabolic profile of tumors, we injected succinate in xenograft tumors. As a control, we also evaluated the effects when PBS and fumarate were injected. Tumor uptake was analyzed by small-animal PET/CT imaging and autoradiography. Intratumoral injections of succinate significantly increased ${ }^{18} \mathrm{~F}$-FDG uptake at $24 \mathrm{~h}$ on smallanimal PET/CT $(P=0.0014, n=3$, Fig. 1A $)$ and autoradiography $(P=0.0124, n=3$, Fig. 1B). Supplemental Video 1 is a video

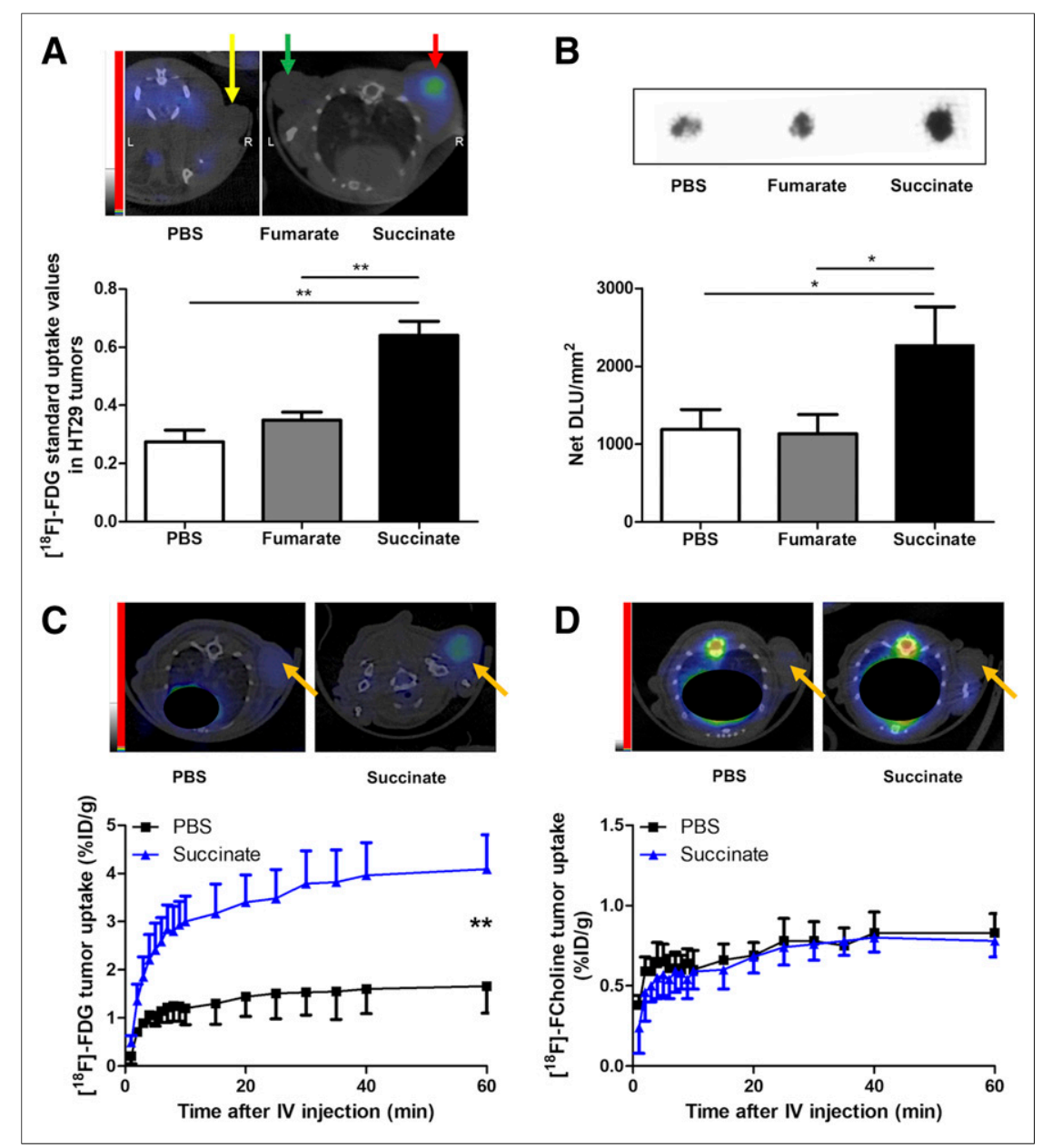

FIGURE 1. (A) Representative PET/CT images of HT-29 tumor-bearing mice $(n=3) 40$ min after ${ }^{18} \mathrm{~F}-\mathrm{FDG}$ injection $(5-10 \mathrm{MBq} / 50 \mu \mathrm{L}$, intraperitoneally) and $27 \mathrm{~h}$ after the first $10-\mu \mathrm{L}$ intratumoral injection of $1 \mathrm{mM}$ succinate (right shoulder, red arrow), $1 \mathrm{mM}$ fumarate (left shoulder, green arrow), or PBS (right hind limb, yellow arrow) every $6 \mathrm{~h}$ for $24 \mathrm{~h}$, along with graph showing quantifications from ${ }^{18}$ F-FDG small-animal PET/CT in tumors. ${ }^{*} P<0.01$, 1-way ANOVA with Bonferroni post hoc test, 3 mice per condition. (B) Representative autoradiographic images of HT-29 tumors extracted from mice immediately after small-animal PET/CT imaging, along with graph showing quantifications from tumor autoradiography. ${ }^{\star} P<0.05,1$-way ANOVA with Bonferroni post hoc test, 3 mice per condition. (C) Representative small-animal PET/CT images of mouse hind limbs $40 \mathrm{~min}$ after ${ }^{18} \mathrm{~F}-\mathrm{FDG}$ injection (5-10 MBq/50 $\mu \mathrm{L}$, intravenously) and $27 \mathrm{~h}$ after first succinate or PBS injection every $6 \mathrm{~h}$ for $24 \mathrm{~h}$, along with graph showing quantifications expressed as percentage injected dose per gram of tissue $(\% \mathrm{ID} / \mathrm{g})$ over time from dynamic small-animal PET/CT reconstruction. ${ }^{* *} P<0.01$, MannWhitney test, 3 mice per condition. (D) Representative small-animal PET/CT images 40 min after ${ }^{18}$ F-fluorocholine injection (5-7 MBq/50 $\mu \mathrm{L}$, intravenously) and $27 \mathrm{~h}$ after first succinate or PBS injection every $6 \mathrm{~h}$ for $24 \mathrm{~h}$, along with graph showing quantifications expressed as \%ID/g over time from dynamic small-animal PET/CT reconstruction. $P=0.609$, Mann-Whitney test, 3 mice per condition. DLU = digital light units. 
recording of the in vivo dynamic ${ }^{18} \mathrm{~F}-\mathrm{FDG}$ uptake after intratumoral injection of $10 \mu \mathrm{L}$ of PBS (top half of video) or 10 $\mu \mathrm{L}$ of a $1 \mathrm{nmol} / \mu \mathrm{L}$ succinate solution (bottom half of video) every $6 \mathrm{~h}$ for $24 \mathrm{~h}$ (supplemental materials are available at http://jnm.snmjournals.org). The limited resolution of autoradiography did not allow us to discriminate the effects of succinate in the different compartments in vivo. To test whether tracer uptake was linked to glucose metabolism and not related to increased blood flow or increased capillary permeability induced by succinate, we performed a head-to-head comparison between ${ }^{18} \mathrm{~F}-\mathrm{FDG}$ and ${ }^{18} \mathrm{~F}$-fluorocholine on dynamic small-animal PET/ CT. Intratumoral injection of succinate induced a significant increase in intratumoral ${ }^{18} \mathrm{~F}$-FDG uptake compared with intratumoral injection of PBS $(P<0.0096, n=3$, Fig. 1C), but no significant change in ${ }^{18} \mathrm{~F}$-fluorocholine uptake was observed in either group $(P=0.6088, n=3$, Fig. 1D).

\section{Succinate Increases ${ }^{18}$ F-FDG Uptake by Endothelial Cells but Not in Tumor Cells or Fibroblasts In Vitro}

We next sought to obtain information on whether tumor or stromal cells could be responsible for our observed metabolic changes. Tumors, endothelial cells, and fibroblasts were treated with varying concentrations of succinate, as well as with PBS and fumarate as controls. To test whether succinate could produce metabolic changes independently of cell density, we analyzed both ${ }^{18}$ F-FDG uptake and cell viability. We found that both the presence of succinate and its concentration significantly influenced ${ }^{18}$ F-FDG uptake by endothelial cells $(P=0.0023, n=3)$. Compared with fumarate, succinate significantly increased ${ }^{18} \mathrm{~F}-\mathrm{FDG}$ uptake by HUVECs at concentrations of $0.1(P=0.0125, n=3)$, $1.0(P=0.0028, n=3)$, and $10 \mathrm{nmol} / \mu \mathrm{L}(P=0008, n=3)$. No significant effect was observed at a $0.01 \mathrm{nmol} / \mu \mathrm{L}$ concentration of succinate (Fig. 2). Succinate slightly, but not significantly, decreased ${ }^{18}$ F-FDG uptake by HT-29 cells and fibroblasts. No matter the cell lineage, the total number of live cells was not significantly affected by the presence of succinate when compared with fumarate and PBS.

\section{Succinate Increases In Vivo ${ }^{18}$ F-FDG Uptake and Retention by Connective Tissue}

To test whether a modification in the uptake pattern of connective tissue could produce the changes on PET imaging, we evaluated the effect of intramuscular injection of succinate in mice. There was a significant increase in ${ }^{18} \mathrm{~F}-\mathrm{FDG}$ uptake by muscle compared with the contralateral muscle injected with either PBS $(P=0.0162, n=3)$ or fumarate $\left(P=0.0458, n=3\right.$, Fig. 3A), but there was no effect on ${ }^{18} \mathrm{~F}$ fluorocholine uptake $(P=0.6173, n=3$, and $P=0.92303, n=3$, respectively, Fig. 3B). Finally, no difference in hind limb blood flow was observed on laser-Doppler $(P=1.000, n=3$, and $P=0.7500$, $n=3$, respectively, Fig. 3C).

\section{GLUT1 Immunohistochemistry}

GLUT1 expression quantification did not significantly differ between study groups in HUVECs or HT-29 tumors (in either epithelial or stromal compartments) (Supplemental Figs. 1 and 2).

\section{DISCUSSION}

It is now evident that succinate should be viewed not only as a metabolite donor in the TCA cycle but also as a signaling molecule with hormonelike functions, which could play a vital role during various pathophysiologic conditions such as ischemia (4) and inflammation (29). The identification of cancer-associated mutations in the TCA cycle enzymes has also highlighted the prevailing notion that aberrant metabolic function can contribute to carcinogenesis via a broad spectrum of pathways such as hypoxic, inflammatory, and immune system responses, as well as epigenetic reprogramming $(30,31)$. Succinate has been shown to stabilize HIF- $\alpha$ via inhibition of prolyl hydroxylases in the cytosol, suggesting a mechanistic link between $S D H x$ mutations, high tumor vascularity, and the ${ }^{18} \mathrm{~F}$-FDG uptake profile in the absence of $V H L$ mutations $(12,32)$. Although this hypothesis is appealing, it should probably be applied to all tumors. Furthermore, the metabolic impact of a malfunctioning TCA is partially compensated
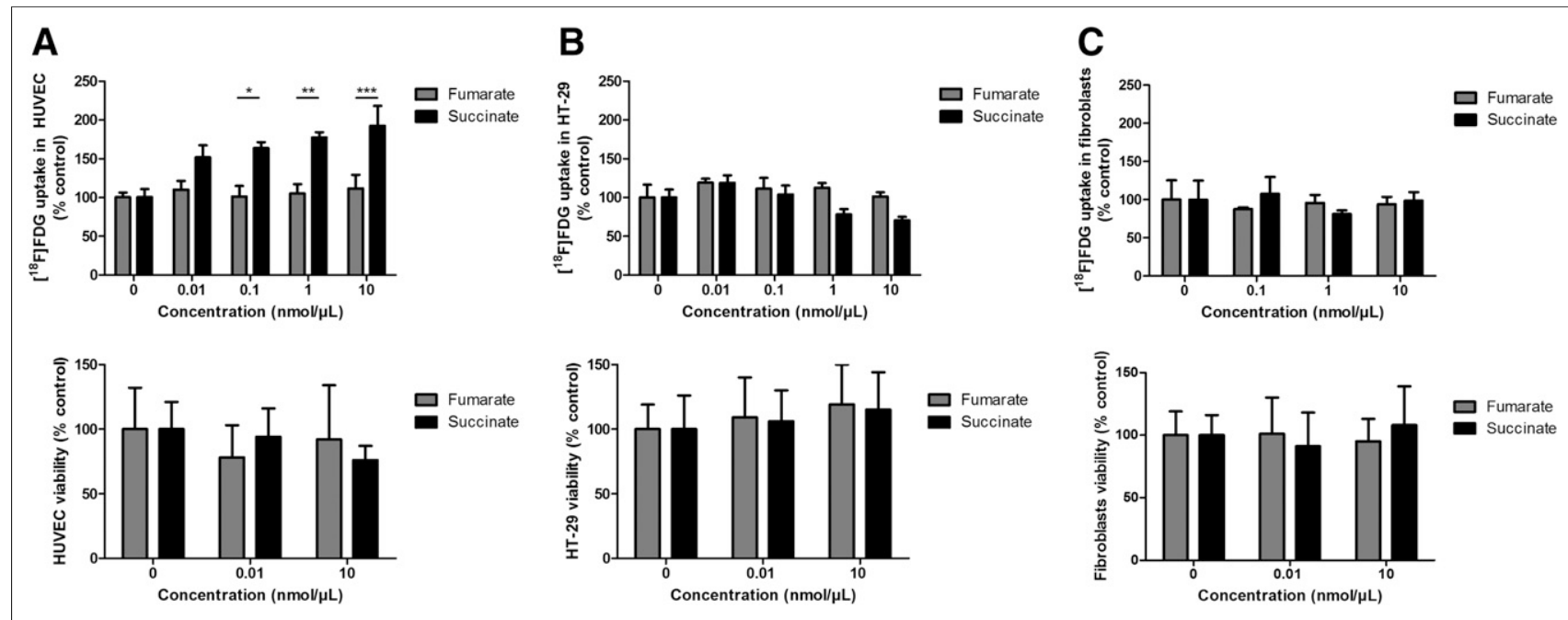

FIGURE 2. Influence of succinate pretreatment on ${ }^{18} \mathrm{~F}-\mathrm{FDG}$ uptake in HUVECs (A, top), in HT-29 cells (B, top), and in primary cardiac fibroblasts (C, top) after pretreatment for $24 \mathrm{~h}$ with $0,0.01,0.1,1.0$, or $10 \mathrm{nmol}$ of succinate per $\mu \mathrm{L}$ of culture medium. ${ }^{\star} P<0.05,{ }^{\star \star} P<0.01$, and ${ }^{\star \star \star} P<0.001,2$-way ANOVA with Bonferroni post hoc test, 3 mice per condition. Viability of HUVECs (A, bottom), HT-29 cells (B, bottom), and fibroblasts (C, bottom) by counting on Kova slides with trypan blue after $24-\mathrm{h}$ incubation with 0.01 or $10 \mathrm{nmol} / \mu \mathrm{L}$ fumarate or succinate, compared with PBS treatment. $P=0.609,0.991$, and 0.715 for HUVECs, HT29, and fibroblasts, respectively, 2-way ANOVA with Bonferroni post hoc test, 3 mice per condition. 

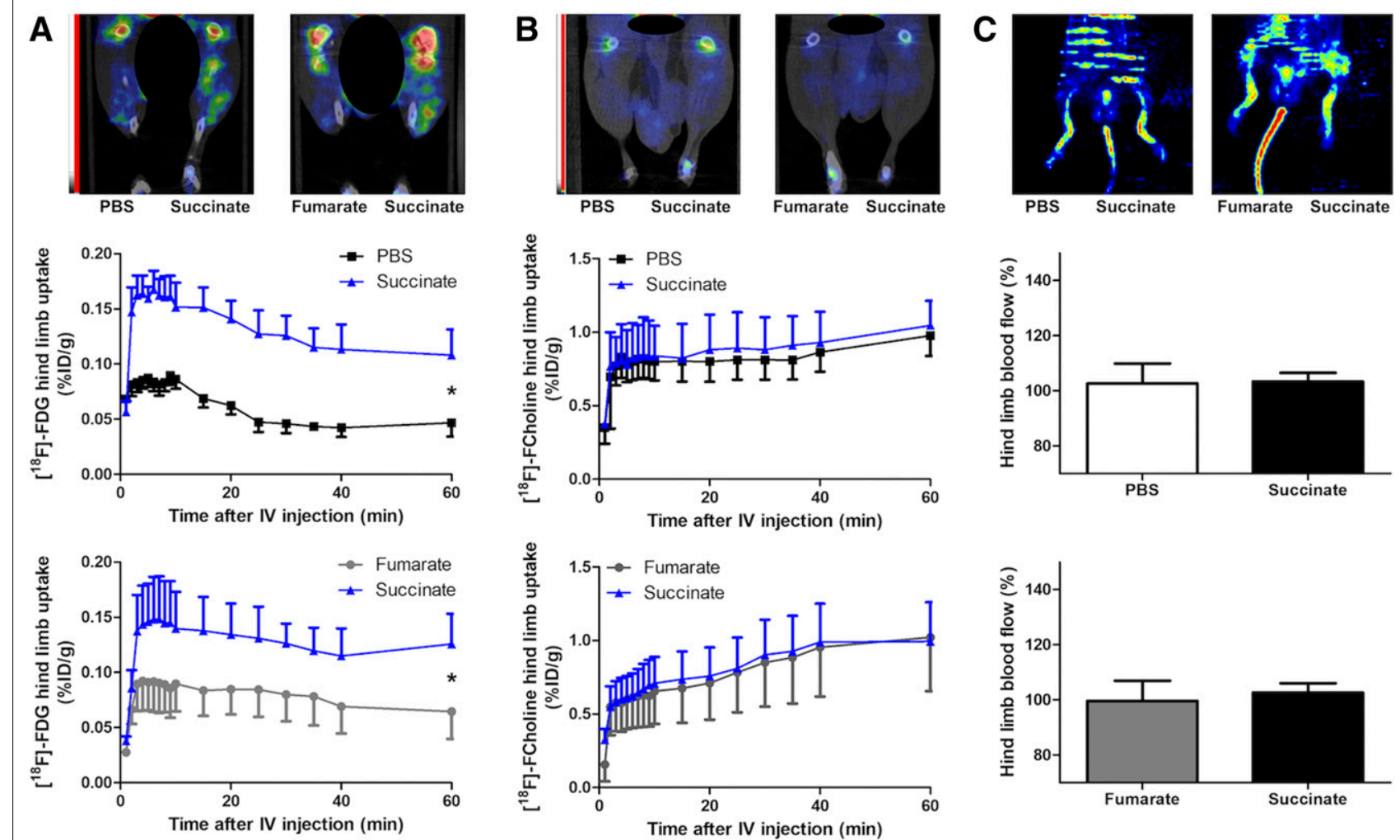

FIGURE 3. (A) Representative small-animal PET/CT images of mouse hind limbs 40 min after ${ }^{18} \mathrm{~F}-\mathrm{FDG}$ injection $(5-10 \mathrm{MBq} / 50 \mu \mathrm{L}$, intravenously) and $27 \mathrm{~h}$ after first succinate (right hind limb) or fumarate or PBS (left hind limb) injection every $6 \mathrm{~h}$ for $24 \mathrm{~h}$, along with graph showing quantifications in each hind limb expressed as percentage injected dose per gram of tissue (\%ID/g) over time from dynamic small-animal PET/CT reconstruction. ${ }^{*} P<0.05$, Mann-Whitney test, 3 mice per condition. (B) Representative small-animal PET/CT images of mouse hind limbs 40 min after ${ }^{18} \mathrm{~F}$ fluorocholine injection (5-7 MBq/50 $\mathrm{LL}$, intravenously) and $27 \mathrm{~h}$ after first succinate (right hind limb) or fumarate or PBS (left hind limb) injection every $6 \mathrm{~h}$ for $24 \mathrm{~h}$, along with graph showing quantifications in each hind limb expressed as \%ID/g over time from dynamic small-animal PET/CT reconstruction. $P=0.617$ vs. PBS and $P=0.923$ vs. fumarate, respectively, not statistically significant, Mann-Whitney test, 3 mice per condition. (C) At top are representative laser-Doppler perfusion images of hind limbs from 6 mice immediately after ${ }^{18}$ F-FDG PET ( $28 \mathrm{~h}$ after first succinate [right hind limb] or fumarate or PBS [left hind limb] injection every $6 \mathrm{~h}$ for $24 \mathrm{~h}$ ). At bottom are corresponding quantifications of perfusion signal in each hind limb.

by the activation of several alternative pathways that can provide metabolites able to enter the TCA at various checkpoints $(15,33)$.

It is well understood that quantification of tumor ${ }^{18} \mathrm{~F}-\mathrm{FDG}$ uptake by PET imaging can be hampered by the contribution of the metabolized ${ }^{18} \mathrm{~F}$-FDG fraction located within stromal cells. Additionally, the unmetabolized component of ${ }^{18} \mathrm{~F}-\mathrm{FDG}$ (in the blood within a tumor, in the intercellular spaces, and within the tumor and stromal cells themselves) can also be far from negligible under certain circumstances. During the past $10 \mathrm{y}$, studies have shown that SDHx-PPGLs exhibit highly elevated ${ }^{18}$ F-FDG uptake. Recently, we have shown in a small series that high SUVs can be observed in PPGL despite relative low $k_{3}$ values (the rate constant for ${ }^{18} \mathrm{~F}$-FDG phosphorylation) compared with malignancies that exhibit high $k_{3}$ values (34). This finding suggests that increased ${ }^{18} \mathrm{~F}-\mathrm{FDG}$ uptake cannot be explained solely by intense tumor cell metabolism and could potentially involve the stromal cells.

The present study shows a new hypothesis based on the effect of succinate on ${ }^{18} \mathrm{~F}$-FDG uptake by endothelial cells, expanding its repertoire of extracellular functions. This could partially explain the ${ }^{18}$ F-FDG uptake pattern observed in PPGLs, which are highly vascularized tumors. We also demonstrated that succinateinduced ${ }^{18}$ F-FDG uptake was not due to increased blood flow or increased capillary permeability, since this phenomenon was not observed after injection of ${ }^{18} \mathrm{~F}$-fluorocholine and no increased blood flow was observed on laser-Doppler. The concentrations used throughout this study $(0-10 \mathrm{nmol} / \mu \mathrm{L})$ were previously identified as appropriate by metabolomics studies that did not distinguish intra- from extracellular succinate components. Many studies have shown that $S D H$-mutated tumors contain enormous amounts of succinate (35-38), which can even be detected by in vivo MR spectroscopy $(39,40)$. It is probable that large amounts of succinate are effluxed by the mutated cells. Analyses of compartmentalized levels of TCA cycle metabolites have revealed that yeast with $s d h \Delta$ mutations may aberrantly efflux succinate from the mitochondria to the cytosol (18). Other studies have even reported that succinate can be excreted into the medium during cultivation of yeast $s d h \Delta$ mutants $(19,20)$. It has been speculated that this retrograde pathway may prevent the potential detrimental effects of succinate excess on nonmitochondrial processes (41). Succinate can migrate through the mitochondrial and plasma membrane via different transport systems such as a succinate-fumarate transporter in the inner 
mitochondrial membrane, porins in the outer mitochondrial membrane, and a sodium-independent anion exchanger in the plasma membrane.

The present study shows that endothelial cells may play an important role in ${ }^{18} \mathrm{~F}$-FDG uptake and, in some tumors, may significantly contribute to a final ${ }^{18}$ F-FDG PET image. This finding will provide incentive to better characterize the molecular mechanisms involved in increased ${ }^{18} \mathrm{~F}-\mathrm{FDG}$ uptake in various tumors, including PPGL TCA cycle defects. Unfortunately, because of the lack of a well-characterized human PPGL cell line, further validation is not yet possible. The present results also suggest that the tumor microenvironment plays an extraordinary role in supplying energy and metabolic fuel for a tumor cell $(42,43)$. Notably, the increased glucose uptake in endothelial cells is not due to increased GLUT1 expression. However, increased GLUT1 expression could be due to the involvement of other glucose transporters or increased activity of intracellular hexokinases (44). Finally, it would be interesting to use GPR91 antagonists or nitric oxide signaling modulation to study the signaling pathway involved in succinate-induced glucose uptake by endothelial cells $(45,46)$. The study of this concept could bring a novel therapeutic approach of starving PPGLs.

\section{CONCLUSION}

The present study shows that succinate stimulates ${ }^{18} \mathrm{~F}-\mathrm{FDG}$ uptake by endothelial cells, a finding that partially explains the ${ }^{18}$ F-FDG metabotype observed in tumors with SDH deficiency. Although this study is an ${ }^{18} \mathrm{~F}-\mathrm{FDG}$-based approach, it provides an impetus to better characterize the determinants of ${ }^{18} \mathrm{~F}-\mathrm{FDG}$ uptake in various tumors and their surrounding microenvironment, with a special emphasis on the role of tumor-specific oncometabolites.

\section{DISCLOSURE}

No potential conflict of interest relevant to this article was reported.

\section{REFERENCES}

1. Brosnan JT, Krebs HA, Williamson DH. Effects of ischaemia on metabolite concentrations in rat liver. Biochem J. 1970;117:91-96.

2. Chouchani ET, Pell VR, Gaude E, et al. Ischaemic accumulation of succinate controls reperfusion injury through mitochondrial ROS. Nature. 2014;515: 431-435.

3. He W, Miao FJ, Lin DC, et al. Citric acid cycle intermediates as ligands for orphan G-protein-coupled receptors. Nature. 2004;429:188-193.

4. de Castro Fonseca M, Aguiar CJ, da Rocha Franco JA, Gingold RN, Leite MF. GPR91: expanding the frontiers of Krebs cycle intermediates. Cell Commun Signal. 2016;14:3.

5. Sapieha P, Sirinyan M, Hamel D, et al. The succinate receptor GPR91 in neurons has a major role in retinal angiogenesis. Nat Med. 2008;14:1067-1076.

6. Hanahan D, Weinberg RA. Hallmarks of cancer: the next generation. Cell. 2011;144:646-674.

7. Letouzé E, Martinelli C, Loriot C, et al. SDH mutations establish a hypermethylator phenotype in paraganglioma. Cancer Cell. 2013;23:739-752.

8. Jochmanova I, Pacak K. Pheochromocytoma: the first metabolic endocrine cancer. Clin Cancer Res. 2016;22:5001-5011.

9. Metellus P, Colin C, Taieb D, et al. IDH mutation status impact on in vivo hypoxia biomarkers expression: new insights from a clinical, nuclear imaging and immunohistochemical study in 33 glioma patients. J Neurooncol. 2011;105: 591-600.

10. Taïeb D, Timmers HJ, Shulkin BL, Pacak K. Renaissance of ${ }^{18}$ F-FDG positron emission tomography in the imaging of pheochromocytoma/paraganglioma. $J$ Clin Endocrinol Metab. 2014;99:2337-2339.
11. Burnichon N, Vescovo L, Amar L, et al. Integrative genomic analysis reveals somatic mutations in pheochromocytoma and paraganglioma. Hum Mol Genet. 2011;20:3974-3985.

12. Favier J, Briere JJ, Burnichon N, et al. The Warburg effect is genetically determined in inherited pheochromocytomas. PLoS One. 2009;4:e7094.

13. López-Jiménez E, Gomez-Lopez G, Leandro-Garcia LJ, et al. Research resource: transcriptional profiling reveals different pseudohypoxic signatures in SDHB and VHL-related pheochromocytomas. Mol Endocrinol. 2010;24: 2382-2391.

14. Pollard PJ, El-Bahrawy M, Poulsom R, et al. Expression of HIF-1alpha, HIF2alpha (EPAS1), and their target genes in paraganglioma and pheochromocytoma with VHL and SDH mutations. J Clin Endocrinol Metab. 2006;91: 4593-4598.

15. Lussey-Lepoutre C, Hollinshead KE, Ludwig C, et al. Loss of succinate dehydrogenase activity results in dependency on pyruvate carboxylation for cellular anabolism. Nat Commun. 2015;6:8784.

16. Rapizzi E, Ercolino T, Fucci R, et al. Succinate dehydrogenase subunit B mutations modify human neuroblastoma cell metabolism and proliferation. Horm Cancer. 2014;5:174-184.

17. Rapizzi E, Fucci R, Giannoni E, et al. Role of microenvironment on neuroblastoma SK-N-AS SDHB-silenced cell metabolism and function. Endocr Relat Cancer. 2015;22:409-417.

18. Lin AP, Anderson SL, Minard KI, McAlister-Henn L. Effects of excess succinate and retrograde control of metabolite accumulation in yeast tricarboxylic cycle mutants. J Biol Chem. 2011;286:33737-33746.

19. Szeto SS, Reinke SN, Sykes BD, Lemire BD. Ubiquinone-binding site mutations in the Saccharomyces cerevisiae succinate dehydrogenase generate superoxide and lead to the accumulation of succinate. J Biol Chem. 2007; 282:27518-27526.

20. Szeto SS, Reinke SN, Sykes BD, Lemire BD. Mutations in the Saccharomyces cerevisiae succinate dehydrogenase result in distinct metabolic phenotypes revealed through ${ }^{1} \mathrm{H}$ NMR-based metabolic footprinting. J Proteome Res. 2010;9:6729-6739.

21. Lendvai N, Pawlosky R, Bullova P, et al. Succinate-to-fumarate ratio as a new metabolic marker to detect the presence of SDHB/D-related paraganglioma: initial experimental and ex vivo findings. Endocrinology. 2014; 155:27-32.

22. Park HJ, Zhang Y, Georgescu SP, Johnson KL, Kong D, Galper JB. Human umbilical vein endothelial cells and human dermal microvascular endothelial cells offer new insights into the relationship between lipid metabolism and angiogenesis. Stem Cell Rev. 2006;2:93-102.

23. Correa de Sampaio P, Auslaender D, Krubasik D, et al. A heterogeneous in vitro three dimensional model of tumour-stroma interactions regulating sprouting angiogenesis. PLoS One. 2012;7:e30753.

24. Haraguchi T, Kayashima T, Okazaki Y, et al. Cecal succinate elevated by some dietary polyphenols may inhibit colon cancer cell proliferation and angiogenesis. J Agric Food Chem. 2014;62:5589-5594.

25. Burt BM, Humm JL, Kooby DA, et al. Using positron emission tomography with $\left[{ }^{18} \mathrm{~F}\right] \mathrm{FDG}$ to predict tumor behavior in experimental colorectal cancer. Neoplasia. 2001;3:189-195.

26. Li XF, Ma Y, Sun X, Humm JL, Ling CC, O’Donoghue JA. High ${ }^{18}$ F-FDG uptake in microscopic peritoneal tumors requires physiologic hypoxia. $\mathrm{J} \mathrm{Nucl}$ Med. 2010;51:632-638.

27. Ahmed D, Eide PW, Eilertsen IA, et al. Epigenetic and genetic features of 24 colon cancer cell lines. Oncogenesis. 2013;2:e71.

28. Bennis Y, Sarlon-Bartoli G, Guillet B, et al. Priming of late endothelial progenitor cells with erythropoietin before transplantation requires the CD131 receptor subunit and enhances their angiogenic potential. J Thromb Haemost. 2012;10:1914-1928.

29. Tannahill GM, Curtis AM, Adamik J, et al. Succinate is an inflammatory signal that induces IL-1beta through HIF-1alpha. Nature. 2013;496:238-242.

30. Ghaffari P, Mardinoglu A, Nielsen J. Cancer metabolism: a modeling perspective. Front Physiol. 2015;6:382.

31. Delgoffe GM, Powell JD. Feeding an army: the metabolism of T cells in activation, anergy, and exhaustion. Mol Immunol. 2015;68(2 pt C):492-496.

32. Selak MA, Armour SM, MacKenzie ED, et al. Succinate links TCA cycle dysfunction to oncogenesis by inhibiting HIF-alpha prolyl hydroxylase. Cancer Cell. 2005; 7:77-85.

33. Zieliński ŁP, Smith AC, Smith AG, Robinson AJ. Metabolic flexibility of mitochondrial respiratory chain disorders predicted by computer modelling. Mitochondrion. 2016;31:45-55.

34. Barbolosi D, Hapdey S, Battini S, et al. Determination of the unmetabolised ${ }^{18} \mathrm{~F}-$ FDG fraction by using an extension of simplified kinetic analysis method: clinical evaluation in paragangliomas. Med Biol Eng Comput. 2016;65:103-111. 
35. Imperiale A, Moussallieh FM, Roche P, et al. Metabolome profiling by HRMAS NMR spectroscopy of pheochromocytomas and paragangliomas detects SDH deficiency: clinical and pathophysiological implications. Neoplasia. 2015;17:55-65.

36. Imperiale A, Moussallieh FM, Sebag F, et al. A new specific succinate-glutamate metabolomic hallmark in SDHx-related paragangliomas. PLoS One. 2013;8: e80539.

37. Richter S, Peitzsch M, Rapizzi E, et al. Krebs cycle metabolite profiling for identification and stratification of pheochromocytomas/paragangliomas due to succinate dehydrogenase deficiency. J Clin Endocrinol Metab. 2014;99:3903-3911.

38. Rao JU, Engelke UF, Sweep FC, et al. Genotype-specific differences in the tumor metabolite profile of pheochromocytoma and paraganglioma using untargeted and targeted metabolomics. J Clin Endocrinol Metab. 2015;100: E214-E222.

39. Varoquaux A, Le Fur Y, Imperiale A, et al. Magnetic resonance spectroscopy of paragangliomas: new insights into in vivo metabolomics. Endocr Relat Cancer. 2015;22:M1-M8.

40. Lussey-Lepoutre C, Bellucci A, Morin A, et al. In vivo detection of succinate by magnetic resonance spectroscopy as a hallmark of SDHx mutations in paraganglioma. Clin Cancer Res. 2016;22:1120-1129.
41. Pollard PJ, Briere JJ, Alam NA, et al. Accumulation of Krebs cycle intermediates and over-expression of HIF1 alpha in tumours which result from germline $\mathrm{FH}$ and SDH mutations. Hum Mol Genet. 2005;14:2231-2239.

42. Gravel SP, Avizonis D, St-Pierre J. Metabolomics analyses of cancer cells in controlled microenvironments. Methods Mol Biol. 2016;1458:273-290.

43. Carito V, Bonuccelli G, Martinez-Outschoorn UE, et al. Metabolic remodeling of the tumor microenvironment: migration stimulating factor (MSF) reprograms myofibroblasts toward lactate production, fueling anabolic tumor growth. Cell Cycle. 2012;11:3403-3414.

44. Tumova S, Kerimi A, Porter KE, Williamson G. Transendothelial glucose transport is not restricted by extracellular hyperglycaemia. Vascul Pharmacol. 2016;87:219-229.

45. Leite LN, Gonzaga NA, Simplicio JA, et al. Pharmacological characterization of the mechanisms underlying the vascular effects of succinate. Eur J Pharmacol. 2016;789:334-343.

46. Paik JY, Lee KH, Ko BH, Choe YS, Choi Y, Kim BT. Nitric oxide stimulates ${ }^{18} \mathrm{~F}$-FDG uptake in human endothelial cells through increased hexokinase activity and GLUT1 expression. J Nucl Med. 2005;46: 365-370. 